\title{
NUMERICAL SOLUTIONS OF IVP USING FINITE ELEMENT METHOD WITH TAYLOR SERIES
}

\author{
Md. Shafiqul Islam ${ }^{1}$, Mostak Ahmed ${ }^{2}$ and M. Alamgir Hossain ${ }^{3}$ \\ ${ }^{1}$ Department of Mathematics \\ University of Dhaka, Dhaka-1000, Bangladesh \\ Email: mdshafiqul@yahoo.com \\ ${ }^{2,3}$ Department of Mathematics, \\ Jagannath University, Dhaka-1100, Bangladesh
}

Received 05.05.09 Accepted 25.04.11

\begin{abstract}
In this paper, we use the integration technique together with the finite element method to approximate the numerical solution of an initial value problem of differential equations. The function of two variables is expanded into Taylor's series up to order two. We exploit GaussLegendre quadrature rules evaluating the integrals arising in the formulation of the present method to get the better accuracy.
\end{abstract}

\section{Introduction}

The initial value problem of ordinary differential equation is of the form $[1,2,3]$

$$
y^{\prime}(x)=f(x, y), \quad x \in[a, b]
$$

with the initial condition

$$
y(a)=y_{0}
$$

The above equations are equivalent to the integral equation

$$
y(x)=y_{0}+\int_{a}^{x} f(t, y) d t .
$$

Instead of solving Equations (1) and (2), we will solve Equation (3). Thus, we have the formula

$$
y_{m+1}=y_{m}+\int_{x_{m}}^{x_{m+1}} f(x, y) d x .
$$

There are many ways approximating the integration of the equation (4). In this paper, by using the Taylor's series approximation, the function under the integration term $f(x, y)$ is to be transformed into the function of a single variable $x$, and then we use the Gauss-Legendre quadrature rule to evaluate the integral, which is the objective of this paper. 


\section{Formulation}

We first discretize the interval $[a, b]$ into $N$ equally spaced such that the mesh points are $x_{m}=a+m h$, for each $m=0,1,2, \ldots, N$.

The common length of the step size is $h=(b-a) / N$. Now we map each subinterval into standard finite element $(-1 \leq \xi \leq 1)$, [4] as

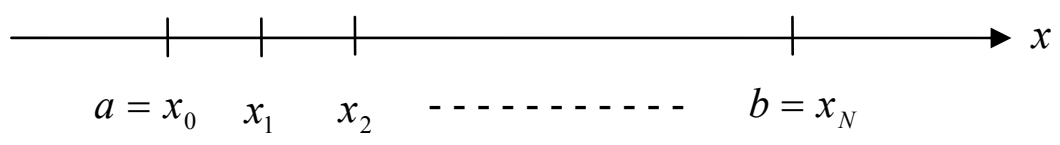

Figure 1: Discretization of the interval $[a, b]$

$x(\xi)=x_{1} L_{1}(\xi)+x_{2} L_{2}(\xi)$

where $L_{1}(\xi)=\frac{1}{2}(1-\xi)$ and $L_{2}(\xi)=\frac{1}{2}(1+\xi)$

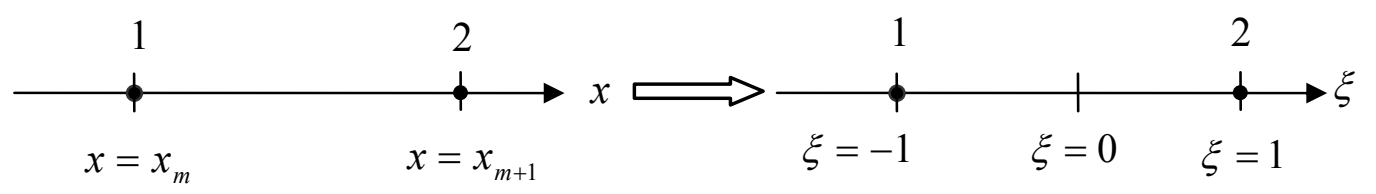

Figure 2: Transformation of arbitrary linear element into equivalent standard linear element

The Gauss Legendre quadrature rule $[1,2]$ is

$$
\int_{a}^{b} f(x) d x \approx \sum_{k=1}^{n} w_{k} f\left(x_{k}\right)
$$

where $x_{k}$ and $w_{k}$ are the Gauss points and the corresponding weight coefficients, respectively.

Now we transform the integral $\int_{x_{m}}^{x_{m+1}} f(x, y) d x$ in the right hand side of (4) into a standard integral using the transformation,

$$
\begin{aligned}
& x(\xi)=\frac{1}{2}\left(x_{m+1}+x_{m}\right)+\frac{\xi}{2}\left(x_{m+1}-x_{m}\right) \\
& d x=\frac{1}{2}\left(x_{m+1}-x_{m}\right) d \xi=\frac{h}{2} d \xi \\
& \text { Then } \int_{x_{m}}^{x_{m+1}} f(x, y) d x=\frac{h}{2} \int_{-1}^{1} f(x(\xi), y(x(\xi))) d \xi
\end{aligned}
$$




$$
=\frac{h}{2} \sum_{i=1}^{n} f\left(x\left(\xi_{i}\right), y\left(x\left(\xi_{i}\right)\right)\right) \text { for } m=0,1,2, \ldots, N .
$$

For each $m, x\left(\xi_{i}\right)=x_{m}+s_{i}$ (say) and $s_{i}$ 's are constants for $i=1,2, \ldots, n$.

To determine $y\left(x\left(\xi_{i}\right)\right)$, we use Taylor's approximation of order two:

$$
y\left(x\left(\xi_{i}\right)\right)=y\left(x_{m}+s_{i}\right)=y_{m}+s_{i} f\left(x_{m}, y_{m}\right)+\frac{s_{i}{ }^{2}}{2}\left[f_{x}\left(x_{m}, y_{m}\right)+f\left(x_{m}, y_{m}\right) f_{y}\left(x_{m}, y_{m}\right)\right]
$$

where $y_{m}=y\left(x_{m}\right)$ and $i=1,2, \ldots, n$.

The equation (4) becomes to

$$
\begin{aligned}
y_{m+1}=y_{m}+\frac{h}{2} \int_{-1}^{1} f(x(\xi), y(x(\xi))) d \xi \\
=y_{m}+\frac{h}{2} \sum_{i=1}^{n} f\left(x\left(\xi_{i}\right), y\left(x\left(\xi_{i}\right)\right)\right)=y_{m}+\frac{h}{2} \sum_{i=1}^{n} w_{i} F\left(\xi_{i}\right) \\
\quad \text { where } F(\xi)=f(x(\xi), y(x(\xi)))
\end{aligned}
$$

\section{Algorithm and Program}

To approximate the solution of the initial-value problem

$$
y^{\prime}=f(x, y), \quad a \leq x \leq b, \quad y(a)=y_{0},
$$

at $(N+1)$ equally spaced numbers in the interval $[a, b]$;

INPUT endpoints $a, b$; initial condition $y_{0}$; order of Gauss-Legendre Quadrature $n$ OUTPUT for some values of $x$ to approximate the corresponding values of $y$.

Step 1 Set $N=(b-a) / h$;

$$
y_{1}=y_{0}
$$

Step 2 For $m=1,2,3, \cdots \cdots \cdots, N+1$ do Step 3

$$
\text { Step } 3 \text { Set } x_{m}=a+(m-1) h \text {; }
$$

Step 4 For $i=1,2,3, \cdots \cdots \cdots, n$ do Step 5

Step 5 Set $\xi_{i}=$ gaussian points

$w_{i}=$ Corresponding weight coefficients

Step 6 For $m=1,2,3, \cdots \cdots \cdots, N$ do Steps 7-11

Step 7 Set sum $=0$;

Step 8 For $k=1,2,3, \cdots \cdots \cdots, n$ do Steps 9-10

$$
\begin{aligned}
\text { Step } 9 \text { Set } x \xi=\frac{1}{2}\left(x_{m+1}+x_{m}\right)+\frac{\xi_{k}}{2}\left(x_{m+1}-x_{m}\right) ; \\
s=x \xi-x_{m} ;
\end{aligned}
$$




$$
y \xi=x_{m}+s f\left(x_{m}, y_{m}\right)+\frac{s^{2}}{2}\left[f_{x}\left(x_{m}, y_{m}\right)+f\left(x_{m}, y_{m}\right) f_{y}\left(x_{m}, y_{m}\right)\right] ;
$$

Step 10 Set $\operatorname{sum}=\operatorname{sum}+w_{k} f(x \xi, y \xi)$;

Step 11 Set $y_{m+1}=y_{m}+\frac{h}{2}$ sum;

Step 12 OUTPUT $\left(x_{i}, y_{i}\right)$.

Step 13 STOP

\section{MathematicaProgram}

$$
\begin{aligned}
& \ln [1]:=\mathbf{f}\left[\mathbf{x}_{-}, \mathbf{Y}_{-}\right]:=\frac{\mathbf{x} \mathbf{Y}^{3}}{\sqrt{1+x^{2}}} ; \\
& \text { DSolve }\left[\left\{Y^{\prime}[x]==\frac{x(y[x])^{3}}{\sqrt{1+x^{2}}}, Y[0]=1\right\}, Y[x], x\right] \\
& \operatorname{Out}[2]=\left\{\left\{\mathrm{Y}[\mathrm{X}] \rightarrow \frac{1}{\sqrt{3-2 \sqrt{1+\mathrm{X}^{2}}}}\right\}\right\} \\
& \ln [3]:=\ll \text { HumericalWath 'Gaussian0uadrature } \\
& \ln [4]:=\mathbf{f x}\left[\mathbf{x}_{-}, \mathbf{Y}_{-}\right]=\mathbf{D}[\mathbf{f}[\mathbf{x}, \mathbf{y}], \mathbf{x}] \text {; } \\
& f_{Y}\left[x_{-}, Y_{-}\right]=D[f[x, Y], Y] \text {; } \\
& y\left[x_{-}\right]:=\frac{1}{\sqrt{3-2 \sqrt{1+x^{2}}}} \text {; }
\end{aligned}
$$




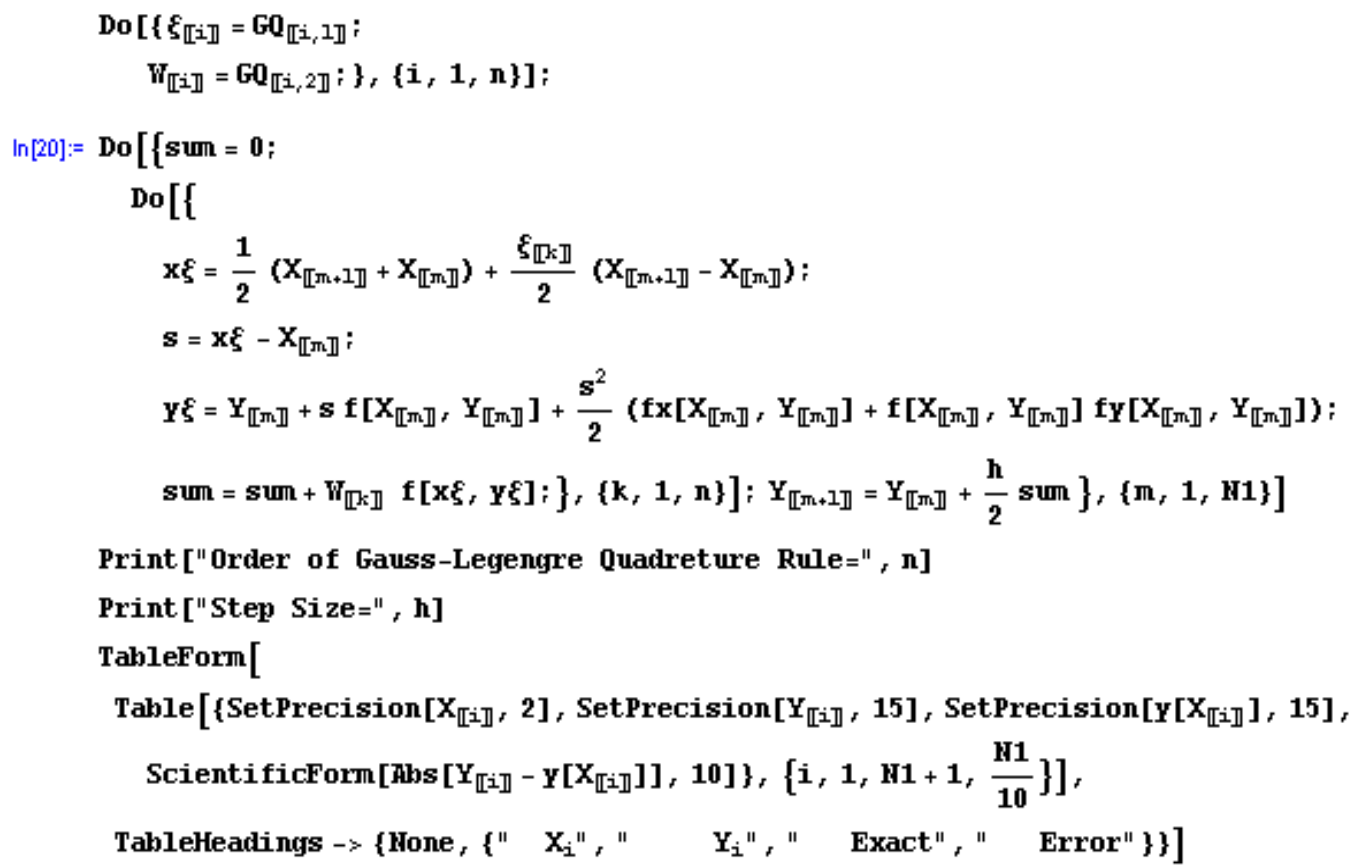

\section{Numerical Examples}

In this section we consider two examples in which Example 1 is taken from [3] and Example 2 is taken from [2] to verify the effectiveness of our formulation presented in the previous section.

Example 1: Consider the numerical solution of the equation [3]

$$
y^{\prime}(x)=\frac{x y^{3}}{\sqrt{1+x^{2}}}, \quad x \in[0,1], \quad y(0)=1
$$

The analytic solution of the above equations is

$$
y(x)=\frac{1}{\sqrt{3-2 \sqrt{1+x^{2}}}} .
$$

Example 2: Consider the numerical solution of the equation [2]

$$
y^{\prime}(x)=\left(x+2 x^{3}\right) y^{3}-x y, \quad 0 \leq x \leq 1, \quad y(0)=\frac{1}{3}
$$

The analytic solution of the above equations is

$$
y(x)=\left(3+2 x^{2}+6 e^{x^{2}}\right)^{-\frac{1}{2}}
$$

Using present formulation for the third order Gauss-Legendre quadrature rule, the approximate solutions are shown in Table 1, which are obtained by the Mathematica 
Program. We also compute the errors with exact solutions by $E=\mid$ Exact-Approx. $\mid$ and are displayed in Table 1.

Table 1: Evaluation of approximate solutions using present formulation

\begin{tabular}{|c|c|c|c|c|}
\hline & \multicolumn{2}{|c|}{ Example 1} & \multicolumn{2}{|c|}{ Example 2} \\
\hline$x$ & (Approx. value) & Error $(E)$ & (Approx. value) & Error $(E)$ \\
\hline \multicolumn{5}{|c|}{ Step size, $h=0.1$} \\
\hline 0 & 1.00000000000000 & 0 & 0.333333333333333 & 0 \\
\hline 0.10 & 1.00502506250022 & $1.261810898 \times 10^{-7}$ & 0.331856160086815 & $4.766246897 \times 10^{-9}$ \\
\hline 0.20 & 1.02041042662246 & $1.883238168 \times 10^{-6}$ & 0.327475502187055 & $6.415010012 \times 10^{-8}$ \\
\hline 0.30 & 1.04716212300636 & $7.842056093 \times 10^{-6}$ & 0.320337922687961 & $2.176400152 \times 10^{-7}$ \\
\hline 0.40 & 1.08723242868709 & $2.338834206 \times 10^{-5}$ & 0.310669391497879 & $4.797366930 \times 10^{-7}$ \\
\hline 0.50 & 1.14406142719502 & $6.137844035 \times 10^{-5}$ & 0.298752615848323 & $8.408279858 \times 10^{-7}$ \\
\hline 0.60 & 1.22371468395705 & $1.561276345 \times 10^{-4}$ & 0.284902989428085 & $1.276412350 \times 10^{-6}$ \\
\hline 0.70 & 1.33745993448850 & $4.133684514 \times 10^{-4}$ & 0.269447658788177 & $1.758875964 \times 10^{-6}$ \\
\hline 0.80 & 1.50846807007518 & $1.234117332 \times 10^{-3}$ & 0.252710609020833 & $2.266745158 \times 10^{-6}$ \\
\hline 0.90 & 1.79342317878687 & $4.733201783 \times 10^{-3}$ & 0.235004691372392 & $2.789187064 \times 10^{-6}$ \\
\hline 1.0 & 2.38 & $3.156623032 \times 10^{-2}$ & 91091 & $3.325897822 \times 10^{-6}$ \\
\hline \multicolumn{5}{|c|}{ Step size, $h=0.01$} \\
\hline 0 & 1.00000000000000 & 0 & 0.333333333333333 & 0 \\
\hline 0.10 & 1.00502518842526 & $2.560505141 \times 10^{-10}$ & 0.331856155329971 & $9.403477996 \times 10^{-12}$ \\
\hline 0.20 & 1.02041230760477 & $2.255855724 \times 10^{-9}$ & 438108880 & $7.192518803 \times 10^{-11}$ \\
\hline 0.30 & 1.04716995613124 & $8.931210882 \times 10^{-9}$ & 0.320337705272432 & $2.244858743 \times 10^{-10}$ \\
\hline 0.40 & 1.08725579035485 & $2.667429633 \times 10^{-8}$ & 0.310668912240318 & $4.791320118 \times 10^{-10}$ \\
\hline 0.50 & 1.14412273431291 & $7.132245705 \times 10^{-8}$ & 0.298751775845289 & $8.249514960 \times 10^{-10}$ \\
\hline 0.60 & 1.22387062449678 & $1.870947743 \times 10^{-7}$ & 714253402 & $1.237666247 \times 10^{-9}$ \\
\hline 0.70 & 1.33787278420842 & $5.187314858 \times 10^{-7}$ & 0.269445901603319 & $1.691106311 \times 10^{-9}$ \\
\hline 0.80 & 1.50970052066639 & $1.666740491 \times 10^{-6}$ & 0.252708344441461 & $2.165785051 \times 10^{-9}$ \\
\hline 0.90 & 1.79814905119406 & $7.329376235 \times 10^{-6}$ & 0.235001904837957 & $2.652628228 \times 10^{-9}$ \\
\hline 1.0 & 2.41414427510185 & $6.928727125 \times 10^{-5}$ & 0.216626423945401 & $3.152131417 \times 10^{-9}$ \\
\hline \multicolumn{5}{|c|}{ Step size, $h=0.001$} \\
\hline 0 & 00000 & 0 & 0.333333333333333 & 0 \\
\hline 0.10 & 1.00502518868105 & $2.584599201 \times 10^{-13}$ & 0.331856155320577 & $9.270362256 \times 10^{-15}$ \\
\hline 0.20 & 1.02041230985836 & $2.269961996 \times 10^{-12}$ & 0.327475438037027 & $7.188694084 \times 10^{-14}$ \\
\hline 0.30 & 1.04716996505345 & $9.000133971 \times 10^{-12}$ & 0.320337705048170 & $2.237099395 \times 10^{-13}$ \\
\hline 0.40 & 1.08725581700220 & $2.694355850 \times 10^{-11}$ & 0.310668911761663 & $4.776734563 \times 10^{-13}$ \\
\hline 0.50 & 1.14412280556312 & $7.224887355 \times 10^{-11}$ & 0.298751775021159 & $8.217870828 \times 10^{-13}$ \\
\hline 0.60 & 1.22387081140135 & $1.902009661 \times 10^{-10}$ & 0.284901713016968 & $1.232070002 \times 10^{-12}$ \\
\hline 0.70 & 1.33787330241000 & $5.298983474 \times 10^{-10}$ & 0.269445899913896 & $1.682931572 \times 10^{-12}$ \\
\hline 0.80 & 1.50970218569168 & $1.715205755 \times 10^{-9}$ & 0.252708342277830 & $2.154609824 \times 10^{-12}$ \\
\hline 0.90 & 1.79815637292522 & $7.645072131 \times 10^{-9}$ & 0.235001902187967 & $2.638028684 \times 10^{-12}$ \\
\hline 1.0 & 2.41421348748317 & $7.488992804 \times 10^{-8}$ & 0.216626420796404 & $3.133854287 \times 10^{-12}$ \\
\hline
\end{tabular}




\begin{tabular}{c|c|c|c|c}
\hline \multicolumn{5}{c}{ Step size, $h=0.0001$} \\
\hline 0 & 1.00000000000000 & 0 & 0.3333333333333333 & 0 \\
0.10 & 1.00502518868131 & $1.332267630 \times 10^{-15}$ & 0.331856155320568 & $3.885780586 \times 10^{-16}$ \\
0.20 & 1.02041230986062 & $5.995204333 \times 10^{-15}$ & 0.327475438036956 & $9.992007222 \times 10^{-16}$ \\
0.30 & 1.04716996506244 & $1.287858709 \times 10^{-14}$ & 0.320337705047948 & $1.332267630 \times 10^{-15}$ \\
0.40 & 1.08725581702911 & $3.308464613 \times 10^{-14}$ & 0.310668911761187 & $1.665334537 \times 10^{-15}$ \\
0.50 & 1.14412280563529 & $7.549516567 \times 10^{-14}$ & 0.298751775020339 & $1.554312234 \times 10^{-15}$ \\
0.60 & 1.22387081159136 & $1.949551631 \times 10^{-13}$ & 0.284901713015738 & $2.331468352 \times 10^{-15}$ \\
0.70 & 1.33787330293937 & $5.342393194 \times 10^{-13}$ & 0.269445899912215 & $2.164934898 \times 10^{-15}$ \\
0.80 & 1.50970218740516 & $1.726396803 \times 10^{-12}$ & 0.252708342275678 & $2.720046410 \times 10^{-15}$ \\
0.90 & 1.79815638056261 & $7.684519687 \times 10^{-12}$ & 0.235001902185332 & $3.330669074 \times 10^{-15}$ \\
1.0 & 2.41421356229761 & $7.548761616 \times 10^{-11}$ & 0.216626420793273 & $3.552713679 \times 10^{-15}$ \\
\hline
\end{tabular}

Now we compare our results with the results in the literature [3] which have been obtained by using various Newton-Cote's formulas. Note that, Podisuk et al [3] strongly recommended that the formulas $(6,9,12,15,18,21,24,27$ and 30) should be used to find the numerical solution of an IVP. In contrast, we observe that our present formula gives more accurate results.

Table 2: Comparison of the present errors with the errors of Reference [3]

\begin{tabular}{|c|c|c|c|}
\hline $\begin{array}{c}\text { Formula of } \\
\text { Reference [3] }\end{array}$ & $\begin{array}{c}x=0.1, \\
h=0.1\end{array}$ & $\begin{array}{c}x=0.1, \\
h=0.0001\end{array}$ & $\begin{array}{c}x=1.0, \\
h=0.0001\end{array}$ \\
\hline 6 & $1.2676962557 \times 10^{-5}$ & $1.8189894035 \times 10^{-12}$ & $1.2563396012 \times 10^{-7}$ \\
\hline 9 & $2.4998819848 \times 10^{-5}$ & $3.0922819860 \times 10^{-11}$ & $1.9134677132 \times 10^{-7}$ \\
\hline 12 & $8.4965516520 \times 10^{-6}$ & $8.5544904493 \times 10^{-12}$ & $9.0301900950 \times 10^{-8}$ \\
\hline 15 & $2.9294220072 \times 10^{-6}$ & $5.4569682106 \times 10^{-12}$ & $2.0128936740 \times 10^{-8}$ \\
\hline 18 & $2.6840044534 \times 10^{-6}$ & $5.4569682106 \times 10^{-12}$ & $2.0114384824 \times 10^{-8}$ \\
\hline 21 & $1.1836842218 \times 10^{-6}$ & $5.4569682106 \times 10^{-12}$ & $2.0128936740 \times 10^{-8}$ \\
\hline 24 & $1.3301713442 \times 10^{-7}$ & $5.4569682106 \times 10^{-12}$ & $2.0128936740 \times 10^{-8}$ \\
\hline 27 & $1.2270877692 \times 10^{-7}$ & $5.4569682106 \times 10^{-12}$ & $2.0125298561 \times 10^{-8}$ \\
\hline 30 & $1.3093116769 \times 10^{-7}$ & $5.4569682106 \times 10^{-12}$ & $2.0121660782 \times 10^{-8}$ \\
\hline \multicolumn{4}{|c|}{ Error in Present Method } \\
\hline & $1.2618108980 \times 10^{-7}$ & $1.3322676296 \times 10^{-15}$ & $7.5487616158 \times 10^{-11}$ \\
\hline
\end{tabular}

\section{Conclusion}

Using integration method and finite element method for solving IVP we observe that the error terms are almost smaller than that of [3]. For simplicity of calculation we use first three terms of Taylor's series, but if we use more terms we can find more accurate results. On the other hand, to perform the numerical integration using finite element method we use two-point or three-point Gaussian quadrature, but if we use more Gaussian quadrature points, we also find more accurate results. 


\section{REFERENCES}

1. Philip J. Davis, Philip Robinowitz, Methods of Numerical Integration, Dover publications Inc., USA, 2007.

2. R.L. Burden, J. Douglas Faires, Numerical Analysis, Seventh Ed., Thomson Learning, 2001.

3. Maitree Podisuk, Ungsana Chundang, Wannaporn Sanprasert. Single Step Formulas and Multi-step Formulas of the Integration Method for Solving the Initial Value Problem of Ordinary Differential Equation, Applied Mathematics and Computation, 190, (2007) 1438 - 1444.

4. P.E. Lewis and J.P. Ward - The Finite Element Method: Principles and Applications, Addison-Wesley Publishing Company, 1991. 